\title{
Atividades extensionistas de educação em saúde bucal para pacientes da terceira idade: relato de experiência
}

Lucas Fernando Oliveira Tomaz Ferraresso*; Sabrina Santana Cassemiro*; Cibele Silva Barbosa*; Lirian Adriana Maria Pereira Silva**; Maria Luiza Hiromi Iwakura Kasai***; Rodrigo Tiossi****; Helion Leao Lino-Junior***; Maura Sassahara Higasi****

\author{
* Cirurgião-Dentista, Universidade Estadual de \\ Londrina \\ ** Técnica em Saúde Bucal, Universidade Estadual de \\ Londrina \\ *** Departamento de Medicina Oral e Odontologia \\ Infantil, Universidade Estadual de Londrina \\ **** Departamento de Odontologia Restauradora, \\ Universidade Estadual de Londrina
}

Recebido: 29/06/2021. Aprovado: 22/09/2021.

\begin{abstract}
RESUMO
Trata-se de um estudo de caráter descritivo, do tipo relato de experiência, que tem por objetivo descrever as atividades extensionistas desenvolvidas pelo projeto "Promoção da Saúde Bucal para Pacientes da Terceira Idade" da Universidade Estadual de Londrina em Pastoral da Pessoa Idosa e Instituições de Longa Permanência para Idosos em Londrina/PR e região. Essas ações foram desempenhadas por estudantes, docentes e servidores nos anos de 2017, 2018 e 2019 por meio de palestras e rodas de conversas sobre alimentação saudável, doenças causadas por próteses e doenças bucais. Além disso, foram realizadas escovação dentária supervisionada individual e coletiva, higienização de próteses, exame físico e autoexame. Em relação aos resultados, observou-se que as atividades possibilitaram identificar a realidade vivenciada dessa população, compartilhar saberes e experiências sobre saúde bucal com informações claras e acessíveis para a comunidade. Adicionalmente, essa oportunidade viabilizou aos estudantes estabelecerem vínculos sociais efetivos, a tomada de decisão, a liderança, proatividade e protagonismo. Favoreceu a formação contextualizada e integrada dos estudantes, com atitudes condizentes para realizar atendimentos e cuidados com a pessoa idosa e seus cuidadores em cenários diversificados. Dessa forma, atividades extensionistas revelaram-se poderosas estratégias para estudantes fortalecerem a formação profissional por meio do trabalho de educação em saúde com idosos e cuidadores.

Descritores: Relações Comunidade-Instituição. Saúde do Idoso. Saúde Bucal. Educação em Saúde.
\end{abstract}




\section{INTRODUÇÃO}

O envelhecimento da população é uma tendência mundial, podendo ser observado por meio da rápida e intensa mudança estrutural da pirâmide populacional ${ }^{1}$. Acredita-se que no Brasil, aproximadamente 30 milhões de pessoas são idosos com 60 anos de idade ou mais, e que esse número chegará em 60 milhões até $2040^{1,2}$. Tais informações convergem para as oportunidades e atividades que deverão ser desenvolvidas para o cuidado e atenção à saúde dessa população, algo desafiador, porém indispensável para o alcance de ações acessíveis e exitosas.

Com o aumento da expectativa de vida, os idosos estão mais susceptíveis ao desenvolvimento de problemas de saúde e de algumas doenças. Nessa idade, frequentemente apresentam hipertensão arterial, diabetes, osteoporose e ansiedade ${ }^{3}$. Manifestam ainda, déficit cognitivo, dificuldade motora e baixa autoestima, o que pode influenciar diretamente na saúde bucal ${ }^{4,5}$ e pode trazer como consequência má higiene bucal, alta prevalência de cárie dentária, doenças periodontais, perdas dentárias e edentulismo ${ }^{6,7}$.

Neste contexto, esses problemas bucais da população geriátrica, podem ser explicados pela perpetuação da equivocada concepção do modelo odontológico curativo-resolutivo e a associação negativa do cirurgião-dentista (CD) como técnico e mutilador ${ }^{6,8,9}$. Além disso, o baixo uso dos serviços odontológicos, recorrendo ao tratamento somente na presença de dor e percepção reabilitadora, destacam tais pensamentos ${ }^{6,8,10}$. Por conseguinte, esses fatores são responsáveis por dificuldades mastigatórias e comprometimento da qualidade de vida, com efeitos diretos no bem-estar geral, na alimentação e na imagem do indivíduo ${ }^{7,11}$. Podem ser considerados independentes os idosos que conseguem tomar suas próprias decisões e realizar as atividades cotidianas sozinhos, e os idosos dependentes dependem de terceiros ou serviços especializados para desempenhar tais funções ${ }^{12}$. O processo de envelhecimento é individual, e está relacionado às perdas de funções cognitivas, físicas, além das perdas relacionadas às questões demográficas e econômicas, que se associam para a ocorrência da incapacidade funcional no idoso. Porém, essas condições não necessariamente limitam os idosos a executarem as suas atividades cotidianas $^{13}$.

Desse modo, idosos que demonstrem essas condições e adversidades para realização de sua higiene, devem ser auxiliados e monitorados por cuidadores, proporcionando assim, cuidado adequado com a saúde bucal ${ }^{14,15}$. O cuidador de idosos (CI) pode ser um familiar, amigo ou uma pessoa contratada ${ }^{16}$, sendo responsável pela atenção ao idoso, auxiliando-o e supervisionandoo com suas atividades básicas e diárias ${ }^{15,16}$. Portanto, o CI, ainda que possua experiências e saberes positivos, necessita ampliar as informações e estratégias que possam orientá-lo para o cuidado e a correta execução de ações relativas à saúde geral e bucal da população idosa.

Diante do exposto, reitera-se o fato de que os direitos sociais da população geriátrica, assegurados por leis, como a $n^{\circ} 10.741$, de que institui o Estatuto do Idoso e os direitos às pessoas com idade igual ou superior a $60 \operatorname{anos}^{17}$, encontram-se ineficientes e com lacunas, exigindo a reorganização dos serviços e programas para a integralidade e consolidação da assistência ao idoso $^{18,19}$. Nesse sentido, além de políticas públicas resolutivas, se faz necessário, o desenvolvimento de atividades preventivas e promotoras de saúde com idosos e seus respectivos cuidadores. Esses indivíduos, necessitam de informações verídicas, de valorização e da realização de ações coerentes com as suas reais necessidades. Carecem, principalmente, de ações de saúde bucal em ambientes alternativos e mais acessíveis, o que pode ser viabilizado por meio da integração ensinoserviço-comunidade quando de fato, aliada a atividades extensionistas (AE). 
Nesse segmento, a Pastoral da Pessoa Idosa (PPI) e a Instituição de Longa Permanência para Idosos (ILPI), configuram-se espaços favoráveis para o desenvolvimento dessas ações de educação em saúde e a consequente integração com a comunidade, de forma coletiva e dialógica. A PPI é uma instituição vinculada à Conferência Nacional dos Bispos do Brasil que promove e valoriza seus membros por meio do processo educativo integrado à sua família ${ }^{20}$. Por sua vez, ILPI são estabelecimentos filantrópicos e/ou privados, de caráter permanente ou temporário, que prestam serviços e cuidados integrais aos idosos ${ }^{21}$. Logo, são ambientes que favorecem a melhora da qualidade de vida, o respeito aos direitos, a interação social e contribuem para o bem estar dessa população idosa ${ }^{20,21}$. Representam, portanto, espaços oportunos para a instituição de ensino superior (IES) fortalecer o seu compromisso com as diversas esferas da sociedade, uma vez que garante o cuidado em saúde para a população geriátrica e CI, e ao mesmo tempo, certifica a sua responsabilidade pela formação integral do estudante.

É fato que as AE são poderosos instrumentos para tais práticas em saúde. A formação do $\mathrm{CD}$, requer, a diversificação dos cenários de ensinoaprendizagem para além da sala de aula ${ }^{22-24}$. Logo, atuar em PPI e ILPI, possibilita o compartilhamento de saberes entre estudantecomunidade e permite, a experiência em realidades distintas do intramuro da IES. Ainda assim, viabiliza a comunidade, a construção de sua autonomia, o fortalecimento identitário e a articulação entre elas, estimulando a troca de experiências $^{24-26}$. Destarte, PPI e ILPI, revelam-se cenários adequados para o trabalho em saúde com a população, pois estimula no estudante, a percepção crítica e o olhar humanizado frente aos verdadeiros problemas encontrados.

Desta forma, PPI, ILPI e IES devem atuar de maneira construtiva e compartilhada, possibilitando a inserção de projetos extensionistas vinculados à Odontologia. Essa integração favorece a obtenção de práticas saudáveis pelos idosos e cuidadores e melhora dos indicadores de saúde e higiene bucal ${ }^{4,27}$. Fortalece, sobretudo, o ensino-aprendizagem ativo por meio da construção e aplicação de conhecimentos científicos coerentes com as necessidades da comunidade.

Dentro desse contexto, o objetivo do presente trabalho consiste em relatar as significativas contribuições de um projeto de extensão na formação universitária por meio de um relato de experiência em ações extensionistas em PPI e ILPI.

\section{RELATO DE EXPERIÊNCIA}

Trata-se de um estudo descritivo, do tipo relato de experiência, desenvolvido a partir da vivência de estudantes em atividades desenvolvidas pelo projeto extensionista intitulado "Promoção da Saúde Bucal para Pacientes da Terceira Idade" da Universidade Estadual de Londrina (UEL). Atualmente, esse projeto envolve os departamentos de Odontologia Restauradora (ODO) e Medicina Oral e Odontologia Infantil (MOOI), além de sua parceria com a Secretaria Municipal de Saúde de Londrina por intermédio da Gerência de Odontologia, vinculada à Diretoria de Atenção Primária à Saúde (DAPS).

O presente relato é sobre o trabalho que foi desenvolvido nos anos letivos de 2017, 2018 e 2019. As atividades foram agendadas antecipadamente e conforme a disponibilidade das próprias instituições. Para tanto, contou com a colaboração de 39 (trinta e nove) estudantes do $1^{\circ}$ ao $5^{\circ}$ ano do Curso de graduação de Odontologia, uma Técnica em Saúde Bucal (TSB), bem como quatro professores do curso.

O público alvo do projeto foi composto por idosos e cuidadores, de ambos os gêneros, com predominância do gênero feminino, totalizando uma média de 433 participantes atendidos nas 15 
Pastorais da Pessoa Idosa e 3 ILPI, localizadas no município de Londrina/PR e região.

As atividades planejadas pelos estudantes e docentes consistiram em: palestras e rodas de conversa com temas variados sobre saúde geral e saúde bucal, escovação dentária supervisionada individual e coletiva, higienização de diversas próteses, realização do exame físico e autoexame. Todas as atividades, foram planejadas e executadas dinamicamente, na tentativa de atrair a atenção, cativar e incentivar a participação de todos os atores envolvidos.

\section{Palestras e Rodas de Conversa}

Para começar as atividades, inicialmente, foram realizadas palestras e rodas de conversa no intuito de estreitar o vínculo entre todos os envolvidos, o que resultou em diálogos enriquecedores, reiterando o interesse dos idosos e cuidadores com a presença da equipe. Nesse momento, possibilitou conhecer o arranjo familiar, estilo de vida, rotinas e diferentes realidades dos indivíduos. Logo, permitiu aos estudantes, entenderem as reais necessidades dos idosos e as suas concepções sobre saúde e higiene bucal, oportunizando o estabelecimento de estratégias para estimulá-los a melhorar hábitos e a mudarem seu cotidiano, corroborando com o relato de Sequeira et al. $(2013)^{28}$.

Nessa atividade, idosos e cuidadores apresentavam-se interessados e receptivos com as informações, relatando suas dúvidas e suas experiências. Assim, foram debatidos temas informativos e educativos relacionados à saúde tais como: alimentação saudável (AS), doenças causadas por próteses e doenças bucais. Nessas palestras e rodas de conversa direcionadas a população idosa, houve a necessidade do uso da linguagem coloquial e orientações diretas e claras, para favorecer a compreensão e consequente incorporação das informações no cotidiano. Além disso, o trabalho com esse grupo, requereu soluções flexíveis e atividades aplicáveis às realidades, evitando assim, a recusa pelos participantes.

Desta forma, as recomendações realizadas sobre AS foram compartilhadas para idosos, cuidadores, acompanhantes e nutricionista da ILPI e, basearam-se no direcionamento e estimulação para o consumo de carnes, alimentos frescos, legumes, frutas, leite e derivados, em consonância com os estudos de Souza et al. (2016) ${ }^{29}$ e Caivano et al. $(2020)^{30}$. Isso se justifica, visto que esses alimentos, têm sido relacionados a menores riscos de câncer, diabetes, doenças cardiovasculares e doença de Alzheimer, enquanto alimentos como açúcar, gordura e ultraprocessados podem promover efeito inverso ${ }^{31,32}$, sendo os idosos e cuidadores aconselhados a limitarem a ingestão desses alimentos.

Segundo Souza et al. $(2016)^{29}$, esses indivíduos devem ser incentivados a práticas de AS, com intuito de mitigar os efeitos da alimentação desequilibrada no estado geral de saúde e na incidência de morbidades ${ }^{29}$. Para isso, demandou o esforço, a dedicação e a escuta ativa entre todos os membros participantes, já que os cenários de atuação possibilitam consolidar esses saberes, melhorar a qualidade de vida e originar resultados positivos.

Outro tema presente na palestra, referiu-se ao uso de próteses dentárias em más condições e as suas possíveis repercussões negativas na cavidade bucal. De acordo com Cueto et al. (2012) ${ }^{33}$, os problemas mais comuns encontrados em idosos usuários de próteses são: estomatite protética, úlcera traumática e candidose $^{33}$. Tais intercorrências, estão associadas diretamente ao uso de próteses mal adaptadas, pobre higienização e a ausência de manutenção $0^{34,35}$. Desta forma, a atuação do projeto consistiu em instruir os idosos e cuidadores sobre a atenção com as próteses dentárias, envolvendo desde a correta utilização, higienização, ajustes e confecção de novas próteses quando necessário. Nesse sentido, foram 
orientados a removerem-as durante a noite, deixando-as mergulhadas em um recipiente com água, permitindo a proteção dos tecidos de suporte, melhor circulação sanguínea e manutenção da saúde dos tecidos. Ainda assim, foram aconselhados a não utilizarem-as por um período superior há 5 anos, embora sabe-se que alguns idosos utilizam por períodos maiores, ratificado com os estudos de Bastos et al. $(2015)^{36}$ e Osmari et al. $(2016)^{37}$.

Por fim, o último assunto debatido com os participantes foi relacionado às doenças bucais da pessoa idosa. Essa população apresenta altos índices de cárie dentária e doença periodontal, sendo um problema considerável e que exige estratégias para o seu controle e a sua diminuição ${ }^{6,38}$. Nesse contexto, dentro da potencialidade do projeto, a conduta adotada consistiu em orientações de instrução de higiene, realização da escovação dentária, redução do uso de cigarro e alimentação saudável.

À vista disso, nessas atividades extensionistas, houve a possibilidade de incentivar práticas saudáveis e conscientizar idosos e cuidadores. Trabalhar educação em saúde com essa população, requereu o planejamento de ações contextualizadas, efetivas e adequadas. Os estudantes passaram a ser os principais responsáveis pela construção dessas ações com voz ativa e protagonismo. Em razão disso, exigiu por parte dos acadêmicos o desenvolvimento da paciência para repetição das informações quando necessário, empatia, conhecimento geral e disposição em servir a comunidade.

De modo geral, durante a $\mathrm{AE}$, a comunidade apresentou-se carente de informações verídicas e declararam até mesmo, que algumas instruções sobre as próteses dentárias, jamais terem sido preconizadas pelo $\mathrm{CD}$. Relataram também, não realizarem assiduamente as informações compartilhadas e que a busca pelo serviço de saúde bucal, ocorre apenas em caso de dor.
Especialmente para os idosos, foi observado que possuíam a autopercepção de seus problemas bucais e corporais, entretanto, detinham a falsa crença de não precisarem de atendimentos nessa idade, em consonância aos achados de Fontes et al. $(2015)^{39}$ e Martins et al. $(2020)^{10}$. O cuidado com a saúde bucal estava associado a função estética e mastigatória, sendo o edentulismo, remetido a certo conformismo e ao processo natural de envelhecimento. Além disso, ainda que os idosos desconhecessem a necessidade da remoção da prótese dentária durante a noite, justificaram o seu uso remetendo a questões estéticas e o constrangimento em dormir sem elas.

Cabe destacar que os cuidadores, muitas vezes os próprios filhos dos idosos, sentiam-se responsáveis por oferecer alimentos saudáveis e realizar as tarefas diárias de higiene geral e bucal. Contudo, salientaram as dificuldades de conciliar horários para execução dessas tarefas, tempo excessivo despendido e a resistência por parte dos idosos para a frequente efetuação de todas as orientações.

\section{Escovação Supervisionada Individual e Coletiva}

Durante a palestra foram compartilhadas informações pertinentes ao público alvo, como orientações de escovação, higienização e proservação das próteses dentárias, a fim de melhorar a saúde bucal dos idosos e aumentar a longevidade de suas próteses. Os idosos foram orientados a realizar a higienização das próteses por meio da combinação dos métodos mecânicos e químicos. Essa associação é a opção eleita para a higienização de próteses devido ao fato de ser acessível, fácil execução e de baixo custo, e age atuando na remoção do biofilme e da preservação das próteses dentárias ${ }^{36,40}$.

Após a primeira etapa de explicações, discussões e visualização da técnica correta de como realizar a escovação dentária e ou de suas respectivas próteses, os idosos e cuidadores 
tiveram a oportunidade de realizarem a sua própria higiene, sendo supervisionados pelos estudantes. Esse momento viabilizou sedimentar na prática, os conhecimentos recém adquiridos e sanar eventuais dúvidas. Inicialmente foram realizadas demonstrações de escovação e uso do fio dental em macromodelos de uma forma lúdica e dinâmica. Em um trabalho realizado por Barbosa e Carvalho $(2020)^{4}$, foram desenvolvidas atividades com idosos institucionalizados e verificaram que o uso do lúdico foi eficaz na promoção da saúde bucal ${ }^{4}$.

Em seguida, foram distribuídos kits de higiene bucal compostos por uma escova de dentes e um dentifrício fluoretado. Posteriormente, foi realizada a escovação supervisionada de forma coletiva, porém, em alguns indivíduos, foi necessária a escovação individual, em decorrência da baixa destreza manual e coordenação motora, que dificulta que seja feita eficiente higienização bucal $^{41,42}$.

Durante as atividades, muitos idosos relataram possuir pouco conhecimento sobre a maneira correta de higienizar a cavidade bucal e a prótese. Outros admitiram ter conhecimento prévio, porém não realizar frequentemente algumas técnicas, principalmente o uso do fio dental e o correto armazenamento da prótese dentária. Os relatos dos participantes evidenciaram que há a necessidade de adotar novos hábitos $\mathrm{e}$ melhorar as práticas de saúde. Logo, esses fatos favorecem e incentivam os idosos a buscarem comportamentos adequados em saúde e ao maior uso dos serviços odontológicos.

A grande maioria dos idosos foi receptiva e aceitou de maneira positiva participar das atividades propostas. No entanto, num momento inicial, alguns participantes ofereceram certa resistência, ocasionada principalmente pela timidez, o que motivou os estudantes a buscarem estratégias para resolver essa adversidade e conseguir convencê-los a participarem. Uma das estratégias utilizadas para solucionar o problema da timidez foi a de, após as atividades coletivas, os estudantes entrarem em contato com esses idosos resistentes de forma individual, a fim de esclarecer suas dúvidas. De uma forma geral, os cuidadores também se mostraram receptivos e bastante interessados em aprender.

A escovação supervisionada experienciada pelos idosos representa um excelente artifício para a fixação da informação. Trata-se de uma ação interativa, lúdica e compartilhada que requer do estudante saberes e a criatividade para motivar e estimular os idosos com suas práticas cotidianas positivas. Além de tudo, esse aprendizado pode ser compartilhado pelos idosos para filhos, amigos e vizinhos, o que favorece $o$ alcance de conhecimento para toda a comunidade.

\section{Autoexame Bucal e Exame Físico}

A última atividade desenvolvida pelos estudantes, consistiu na orientação quanto ao autoexame e a realização do exame físico da boca. Esses exames são práticas que buscam um diagnóstico precoce e a prevenção de doenças, possibilitando maiores chances de tratamento, cura e qualidade de vida ${ }^{43,44}$. A importância da execução está no fato de que a população idosa pode apresentar lesões bucais como exposto pelo estudo clinicopatológico de Torres, Sbegue e Costa $(2016)^{45}$, os quais verificaram que as lesões inflamatórias/reativas eram mais comuns, seguido pelas neoplasias malignas com destaque para o carcinoma espinocelular ${ }^{45}$. Portanto, cabe ao CD a realização do exame físico minucioso, bem como informar ao paciente sobre a realização do autoexame ${ }^{46}$.

$\mathrm{Na}$ orientação do autoexame, buscou-se sempre utilizar linguagem compatível com o público com explicações de forma mais didática possível, auxiliadas por figuras ilustrativas que foram apresentadas utilizando o programa PowerPoint ${ }^{\circledR}$ (Microsoft, Redmond, WA, EUA) bem como demonstrações do procedimento. Ao 
final, recebemos muitos questionamentos quanto às lesões presentes na cavidade bucal, a maioria das pessoas relataram nunca ter recebido informações sobre o autoexame, no entanto, mostraram bastante interesse no assunto e participaram ativamente da prática.

Em seguida, o exame físico foi efetuado pelos estudantes que já tiveram a disciplina de estomatologia na academia sob a supervisão do docente responsável com o objetivo de identificar alterações e necessidades odontológicas. Para o exame, foram utilizados abaixador de língua, gazes e a lanterna do celular. Em duplas, os estudantes realizaram o exame físico, enquanto um iluminava o campo o outro examinava de forma sistemática a face, pescoço e a cavidade oral do idoso sentado, por meio das manobras de inspeção, palpação, auscultação e olfato como preconizado por Rados e Rösing $(2014)^{47}$.

Foi possível notar durante o exame, que muitos idosos possuíam alguma necessidade odontológica, seja esta protética, endodôntica, restauradora, entre outras, sendo estomatite protética a lesão mais encontrada, visto que muitos declararam que há anos não faziam manutenção e não removiam a prótese para dormir, corroborando com os estudos de Rosendo et al. (2017) ${ }^{48}$ e Marra et al. $(2017)^{49}$, os quais descreveram que a estomatite protética causada por fungo da Candida albicans está fortemente associada à má higienização, a falta de manutenção, além do uso contínuo dessas próteses ${ }^{48,49}$. Para todas as ILPI envolvidas, diante desses e de outros casos que demandaram cuidados especializados odontológicos, foi realizado o encaminhamento para a Clínica Odontológica Universitária (COU) da UEL, onde estudantes sob supervisão dos professores realizam a assistência.

Os pacientes de urgência e emergência como casos de dor dentária, abscessos e infecções dentárias foram orientados a procurar o Pronto Socorro Odontológico (PSO) da COU-UEL. O serviço foi realizado respeitando a ordem de chegada no estabelecimento, não necessitando do agendamento prévio. Quando verificado a presença de lesões bucais, o estudante realizou $\mathrm{o}$ agendamento para o projeto de extensão “Ambulatório de estomatologia: diagnóstico e tratamento de doenças da cavidade bucal" que ocorreu toda semana nos dias de quarta-feira e sexta-feira.

Para pacientes que necessitavam de tratamento convencional como troca de próteses, restaurações, tratamento periodontal e endodôntico entre outros procedimentos, foram marcados para a disciplina de Clínica Integrada de Odontogeriatria de acordo com a urgência de reabilitação de cada caso. Esses pacientes também foram orientados a procurar a unidade básica de Saúde (UBS) para realizar o agendamento caso houvesse demora em ser chamados na COU-UEL, visto que há uma lista de espera. Desta forma, quando agendados pela UBS o paciente também poderia ser encaminhado para o Centro de Especialidades Odontológicas (CEO).

Foi observado que as ações contribuíram para a formação dos estudantes que desenvolveram a prática no reconhecimento de lesões com maior percepção da realidade dos indivíduos, além do grande enriquecimento pessoal com a interação com o público e a construção de novos conhecimentos. Os idosos e cuidadores, por sua vez, se mostraram motivados em realizar o autoexame e a procurar atendimento. No estudo de Oliveira et $a .^{50}$, os idosos que executavam o autoexame usavam os serviços odontológicos com maior frequência devido à percepção da necessidade de assistência profissional ${ }^{50}$.

A formação profissional durante a graduação em odontologia, deve valorizar o desenvolvimento de ações educativas/preventivas ao grupo de idosos, bem como estratégias de promoção de saúde, de forma a promover um processo de ensino e aprendizagem ao estudante que irá desenvolver 
competências para melhor atendimento a esse grupo populacional alinhadas com as Diretrizes Curriculares Nacionais ${ }^{51}$.

\section{CONSIDERAÇÕES FINAIS}

Destarte, o trabalho em saúde nos ambientes de PPI e ILPI executado em atividades extensionistas, revelou-se estratégia poderosa para promover atenção à saúde para idosos e seus cuidadores. Esses cenários de atuação, permitiram familiarizar o estudante com as demandas sociais dessa população e concomitantemente, incentivar os membros participantes a melhorarem seus hábitos bucais diários e potencializarem práticas saudáveis em saúde.

Ainda assim, as experiências vivenciadas e as propostas de ações efetuadas neste projeto de extensão viabilizaram a aproximação entre o binômio estudante-idoso e estudante-cuidador, interação essa multifacetada e desafiadora, visto que demandou respostas cotidianas positivas e efetivas. Desse modo, essa relação favoreceu a resolução de uma série de dúvidas e esclarecimento de mitos, além do compartilhamento de saberes que muitas vezes, os idosos e cuidadores não possuíam.

Nesse contexto, tem-se em PPI e ILPI cenários oportunos para o desenvolvimento de ações preventivas e motivadoras de educação em saúde, impactando diretamente na valorização social do idoso, na autonomia e qualidade de vida. Desta forma, essas atividades extensionistas devem ser ampliadas e potencializadas nos próximos anos, tendo em vista o panorama do envelhecimento e a necessidade de ações acessíveis e adequadas à população brasileira.

\section{ABSTRACT}

Extension activities in oral health education for elderly patients: an experience report

This is a descriptive study of the experience report type with the purpose of describing the extension actions developed by the project "Promotion of Oral Health for Elderly Patients" developed by the State University of Londrina at the Pastoral of the Elderly and Long-Stay Institutions for the Elderly in the city of Londrina/PR and region. These actions were developed by students, professors, and civil servants in 2017, 2018, and 2019 through lectures and talk sessions on healthy eating habits, diseases caused by prosthetics, and oral diseases. Additionally, supervised individual and collective toothbrushing, cleaning of prosthetics, physical examination and self-examination were developed. In relation to the results, it was observed that the activities allowed the students to identify the reality of that population, sharing knowledges and experiences on oral health with clear and accessible information for the community. Furthermore, this opportunity allowed the students to establish effective social bonds, improve their decision-making process, leadership, proactivity, and protagonism. It also favored the contextualized and integral training of the students, with befitting attitudes to provide care for the elderly and their caregivers in diversified scenarios. Therefore, the extension activities were powerful strategies for the students to strengthen the professional training through health education work with the elderly and their caregivers.

Descriptors: Community-Institution Relationships. Health of the Elderly. Oral Health. Health Education.

\section{REFERÊNCIAS}

1. Instituto Brasileiro de Geografia e Estatística (2018). Projeção da população do Brasil e das Unidades da Federação, revisão 2018. Rio de Janeiro: IBGE. [Acesso em 29 jun. 2021]. Disponível em: https://biblioteca.ibge. gov.br/visualizacao/livros/liv101597.pdf.

2. Instituto Brasileiro de Geografia e Estatística (2016). Síntese de indicadores sociais: uma análise das condições de vida da população brasileira: 2016. Rio de Janeiro: IBGE. [Acesso em 29 jun. 2021]. Disponível em: https://biblioteca.ibge.gov.br/visualizacao/li vros/liv101629.pdf. 
3. Pereira DS, Nogueira JAD, Silva CABD. Qualidade de vida e situação de saúde de idosos: um estudo de base populacional no Sertão Central do Ceará. Rev Bras Geriatr Gerontol. 2015; 18(4):893-908.

4. Barbosa LL, Carvalho RF de. O enfoque lúdico nas intervenções educativas em saúde bucal para idosos institucionalizados. Rev Bras de Ext Univ. 2020; 11(2):189-97.

5. Mariano PP, Carreira L, Lucena ACRM, Salci MA. Desenvolvimento de atividades de estímulo cognitivo e motor: perspectiva de idosos institucionalizados. Esc Anna Nery. 2020; 24(3): e20190265.

6. Brasil. Ministério da Saúde. Secretaria de Atenção à Saúde. Secretaria de Vigilância em Saúde. SB Brasil 2010: Pesquisa Nacional de Saúde Bucal: resultados principais / Ministério da Saúde. Secretaria de Atenção à Saúde. Secretaria de Vigilância em Saúde. -Brasília: Ministério da Saúde, 2012. [Acesso em 29 jun. 2021]. Disponível em: https://bvsms.saude.gov.br/bvs/publicac oes/pesquisa_nacional_saude_bucal.pdf.

7. Gil-Montoya JA, de Mello AL, Barrios R, Gonzalez-Moles MA, Bravo M. Oral health in the elderly patient and its impact on general well-being: a nonsystematic review. Clin Interv Aging. 2015; 11(10):461-7.

8. Brasil. Ministério da Saúde, Secretaria de Atenção à Saúde, Departamento de Atenção Básica, Coordenação-Geral de Saúde Bucal. Passo a passo das Ações da Política Nacional de Saúde Bucal (2016), Brasília. [Acesso em 29 jun. 2021]. Disponível em: https://bvsms.saude.gov.br/bvs/publicacoes/ saude bucal_sistema unico_saude.pdf.

9. Bortoli FR, Moreira MA, Moretti-Pires RO, Botazzo C, Kovaleski DF. Percepção da saúde bucal em mulheres com perdas dentárias extensas. Saúde Soc. 2017; 26(2):533-44.
10. Martins AME de BL, Oliveira RFR de, Haikal DS, Santos ASF, Souza JGS, Alecrim BPA et al. Uso de serviços odontológicos públicos entre idosos brasileiros: uma análise multinível. Ciênc Saúde Coletiva. 2020; 25(6):2113-26.

11. Petry J, Lopes AC, Cassol K. Autopercepção das condições alimentares de idosos usuários de prótese dentária. CoDAS. 2019; 31(3):e20180080.

12. Brasil. Ministério da Saúde. Secretaria de Atenção à Saúde. Departamento de Atenção Básica. Envelhecimento e saúde da pessoa idosa. 1. ed. Cadernos de Atenção Básica, Brasília, DF, n. 19, 2006. 192p. (Série A, Normas e Manuais Técnicos). [Acesso em 29 jun. 2021]. Disponível em: https://bvsms.saude.gov.br/bvs/publicacoes/ evelhecimento_saude_pessoa_idosa.pdf.

13. Mattos ED. Capacidade funcional de idosos dependentes cadastrados na estratégia de saúde da família do município de Londrina/PR. Botucatu. Tese [Doutorado em Saúde Coletiva] - Universidade Estadual Paulista "Júlio de Mesquita Filho"; 2015.

14. Bonfá K, Mestriner SF, Fumagalli IHT, Mesquita LPD, Bulgarelli AF.Percepção de cuidadores de idosos sobre saúde bucal na atenção domiciliar. Rev Bras Geriatr Gerontol. 2017; 20(5):650-9.

15. Oliveira CS, Soares MSM, Vasconcelos SC, Dalle-Piagge CSL, Silva ÂMK, Cabral AG et al. Oral hygiene routine of functionally dependent elderly individuals performed by home caregivers. Int Arch Med. 2017; 9:1755-7682.

16. De Valle-Alonso MJ, Hernández-López IE, Zúñiga-Vargas ML, Martínez-Aguilera P. Sobrecarga y Burnout en cuidadores informales del adulto mayor. Enferm Univ. 2015; 12(1):19-27.

17. Brasil. Lei n. 10.741 , de $1^{\circ}$ de outubro de 
2003. Dispõe sobre o Estatuto do Idoso e dá outras providências. Diário Oficial da União, Brasília, 3 out. 2003. Seção 1, p. 1. [Acesso em 29 jun. 2021]. Disponivel em: http://www.planalto.gov.br/ccivil_03/leis/2 003/110.741.htm.

18. Fernandes MT de O, Soares SM. O desenvolvimento de políticas públicas de atenção ao idoso no Brasil. Rev Esc Enferm USP. 2012; 46(6):1494-502.

19. Romero DE, Pires DC, Marques A, Muzy J. Diretrizes e indicadores de acompanhamento das políticas de proteção à saúde da pessoa idosa no Brasil. RECIIS Rev Eletr Comun, Inf Inov Saúde. 2019; 13(1):134-57.

20. Conferência Nacional dos Bispos do Brasil Regional Oeste 2 [homepage na internet]. Pastoral da Pessoa Idosa. [Acesso em 29 jun. 2021]. Disponível em: https://www. cnbbo2.org.br/pastorais/pastoral-da-pessoaidosa.

21. Barbosa L de M, Noronha K, Camargos MCS, Machado CJ. Perfis de integração social entre idosos institucionalizados não frágeis no município de Natal, Rio Grande do Norte, Brasil. Ciênc Saúde Coletiva. 2020; 25(6):2017-30.

22. Baldoino AS, Veras RM. Análise das atividades de integração ensino-serviço desenvolvidas nos cursos de saúde da Universidade Federal da Bahia. Rev Esc Enferm USP. 2016; 50(spe):17-24.

23. Codato LAB, Garanhani ML, González AD, Carcereri D L, Carvalho BG, Morita MC. Significados do estágio em Unidades Básicas de Saúde para estudantes de graduação. Rev ABENO. 2019; 19(1):2-9.

24. Ferraresso LFOT, Codato LAB. Aprendizados e reflexões advindos de atividade extensionista de educação em saúde em Centros de Educação Infantil. Rev Ciênc Plur, 2021; 7(2):132-48.
25. Gonçalves BD, Saadallah MM, Queiroz IS de. Articulando redes, fortalecendo comunidades: intervenção psicossocial e articulação entre universidade, comunidades e políticas públicas. Pesqui Prát Psicossociais. 2015; 10(1):88-100.

26. Siqueira SMC, Jesus VS de, Santos ENB dos, Whitaker MCO, Sousa BVN, Camargo CL de. Atividades extensionistas, promoção da saúde e desenvolvimento sustentável: experiência de um grupo de pesquisa em enfermagem. Esc Anna Nery. 2017; 21(1):e20170021.

27. Lago JD, Fais LMG, Montandon AAB, Pinelli LAP. Educational program in oral health for caregivers on the oral hygiene of dependent elders. Rev Odontol UNESP. 2017; 46(5):284-91.

28. Sequeira É, Chao RS, Moraes MJT, Haddad, VK, Wen CL. Modelo de teleducação sobre cuidados com a saúde oral do idoso usuário de prótese total dental. Rev ABENO. 2013; 13(2):2-12.

29. Souza JD, Martins MV, Franco FS, Martinho KO, Tinôco AL. Dietary patterns of the elderly: characteristics and association with socioeconomic aspects. Rev Bras Geriatr Gerontol. 2016; 19(6):970-7.

30. Caivano S dos A, Domene SMA. Consensus among experts on healthy eating and diet quality index. Ciênc Saúde Coletiva. 2020; 25(7):2551-60.

31. Louzada MLDC, Martins APB, Canella DS, Baraldi LG, Levy RB, Claro RM et al. Impact of ultra-processed foods on micronutrient content in the Brazilian diet. Rev Saúde Públ. 2015; 49(00):45.

32. Dourado DAQS, Marucci $M$ de FN, Roediger M de A, Duarte YA de O. Padrões alimentares de indivíduos idosos do município de São Paulo: evidências do estudo SABE (Saúde, Bem-estar e 
Envelhecimento). Rev Bras Geriatr Gerontol. 2018; 21(6):731-42.

33. Cueto A, Martínez R, Niklander S, Deichler J, Barraza A, Esguep A. Prevalence of oral mucosal lesions in an elderly population in the city of Valparaiso, Chile. Gerodontology. 2012; 30(3):201-6.

34. Martins AME de BL, Souza JGS, Haikal DS, Paula AMB de, Ferreira EF, Pordeus IA. Prevalência de autoexame bucal é maior entre idosos assistidos no Sistema Único de Saúde: inquérito domiciliar. Ciênc Saúde Coletiva. 2015; 20(4):1085-98.

35. Palati S, Ramani P, Shrelin HJ, Sukumaran G, Ramasubramanian A, Don KR et al. Knowledge, Attitude and practice survey on the perspective of oral lesions and dental health in geriatric patients residing in old age homes. Indian J Dent Res. 2020; 31(1):22-5.

36. Bastos PL, Mesquita TC, Ottoboni GS, Figueiredo VMG. Métodos de higienização em próteses dentais removíveis: Uma revisão de literatura. J Dent Public Heal. 2015; 6(2):129-37.

37. Osmari D, Fraga S, Braun KO, Unfer B. Behaviour of the elderly with regard to hygiene procedures for and maintenance of removable dentures. Oral Health Prev Dent. 2016; 14(1):21-6.

38. López R, Smith PC, Göstemeyer G, Schwendicke F. Ageing, dental caries and periodontal diseases. J Clin Periodontol. 2017; 44:S145-52.

39. Fontes AP, Neri AL. Resilience in aging: literature review. Ciênc Saúde Coletiva. 2015; 20(5):1475-95.

40. Cinquanta L, Varoni EM, Barbieri C, Sardella A. Patient attitude and habits regarding removable denture home hygiene and correlation with prosthesis cleanliness: A cross-sectional study of elderly Italians. J Prosthet Dent. 2021; 125(5):e772.
41. Presa SL. Saúde bucal na terceira idade. Revista Uningá. 2014; 39(1):137-48.

42. Grönbeck Lindén I, Hägglin C, Gahnberg L, Andersson P. Factors Affecting Older Persons' Ability to Manage Oral Hygiene: A Qualitative Study. JDR Clin Trans Res. 2017; 2(3):223-32.

43. Cartaxo AC, Silva DNA, Costa KCAD, Souza GCA, Martins ARLA. Conhecimento de trabalhadores rurais de um município do nordeste brasileiro acerca da prevenção e diagnóstico precoce do câncer de boca. Rev Ciênc Plur. 2017; 3(1):51-62.

44. Silva MA, Souza JR, Oliveira MG, Rocha DAP. Câncer de boca: ação educativa centrada na capacitação para o autoexame. Rev Ciênc Ext. 2018; 14(1):116-24.

45. Torres VST, Sbegue A, Costa CBC. A importância do diagnóstico precoce de câncer bucal em idosos. Rev Soc Bras Clin Med. 2016; 14(1):57-62.

46. Silva BLR da, Silva Neto M dos S da, França DCC, Aguiar SMHC Ávila. Perfil dos participantes do programa permanente de prevenção e diagnóstico precoce das doenças bucais, com ênfase no câncer de boca, no município de Cuiabá-MT. Arch Health Invest. 2017; 6(3):141-4.

47. Rados PV, Rösing CK. Exame das mucosas: uma responsabilidade profissional que pode salvar vidas. Clín Int J Braz Dent. 2014; 10(2):226-9.

48. Rosendo RA, Sousa JNL, Abrantes JGS, Cavalcante ABP, Ferreira AKTF. Autopercepção de saúde bucal e seu impacto na qualidade de vida em idosos: uma revisão de literatura. Rev Saúde Ciênc. 2017; 6(1):89-102.

49. Marra J, Perez LEC, Henriques TE, Pinheiro MT, Castro FLA. Avaliação da correlação entre o grau de instruções e qualidade de higiene de usuários de próteses totais com a 
presença de estomatite protética. Rev Correspondência para:

Odontol Bras Central. 2017; 26(76):15-20. Lucas Fernando Oliveira Tomaz Ferraresso

50. Oliveira RFR, Souza JGS, Haikal DSA, e-mail: lucas.fernando@uel.br

Ferreira EF, Martins AMEBL. Equidade no Clínica Odontológica Universitária

uso de serviços odontológicos provenientes Rodovia Celso Garcia Cid, PR 445, Km 380, s/n

do SUS entre idosos: estudo de base Campus Universitário

populacional. Ciênc Saúde Coletiva. 2016; 86044-766 Londrina/PR 21(11):3509-23.

51. Núñez MDRR, Martini JG, Siedler MJ, Mello ALSFD. O ensino da odontogeriatria e as diretrizes curriculares nos cursos de graduação em odontologia em países da América do Sul. Rev Bras Geriatr Gerontol. 2016; 20(6): 826-835. 